\title{
Article \\ Polarization-Insensitive Unit Cells for a Cost-Effective Design of a 3-D-Printed Fresnel-Lens Antenna
}

\author{
Salvador Moreno-Rodríguez ${ }^{1}$, Miguel A. Balmaseda-Márquez ${ }^{1}$, Javier Carmona-Murillo ${ }^{2}$ (D) \\ and Ángel Palomares-Caballero ${ }^{1, *(\mathbb{D})}$
}

1 Department of Signal Theory, Telematics and Communications, University of Granada (UGR), 18071 Granada, Spain; salvamr96@ugr.es (S.M.-R.); migbalmar@correo.ugr.es (M.A.B.-M.)

2 Department of Computing and Telematics Engineering, University of Extremadura (UEx), 06006 Badajoz, Spain; jcarmur@unex.es

* Correspondence: angelpc@ugr.es

check for

updates

Citation: Moreno-Rodríguez, S.;

Balmaseda-Márquez, M.A.; CarmonaMurillo, J.; Palomares-Caballero, Á. Polarization-Insensitive Unit Cells for a Cost-Effective Design of a

3-D-Printed Fresnel-Lens Antenna. Electronics 2022, 11, 338.

https://doi.org/10.3390/ electronics11030338

Academic Editor: Reza K. Amineh

Received: 28 December 2021

Accepted: 21 January 2022

Published: 23 January 2022

Publisher's Note: MDPI stays neutral with regard to jurisdictional claims in published maps and institutional affiliations.

Copyright: (C) 2022 by the authors. Licensee MDPI, Basel, Switzerland. This article is an open access article distributed under the terms and conditions of the Creative Commons Attribution (CC BY) license (https:/ / creativecommons.org/licenses/by/ $4.0 /)$.

\begin{abstract}
A 3-D printed Fresnel-lens antenna formed by dielectric unit cells insensitive to polarization is presented in this article. The proposed unit cell can be implemented in any azimuth orientation, simplifying the design and the implementation of the Fresnel subzones, which is an advantage over the previous 3-D-printed Fresnel-lens designs. The unit cell exhibits a T-shaped geometry capable of providing no change in relative permittivity under TE polarizations orthogonal to each other. The novel design of the unit cell also provides robustness under oblique incidence and frequency. These features allow the radial arrangement of the unit cells to configure the subzones of the Fresnel lens, ensuring the desired relative permittivity. Additionally, the geometry of the printed unit cells enables self-supported subzones with the minimum number of unit cells per subzone. A 3-Dprinted prototype of the proposed Fresnel lens was manufactured by stereolithography (SLA). The measurement results showed a good agreement with the simulated ones. The measured gain was $26.5 \pm 0.5 \mathrm{dBi}$ from $55 \mathrm{GHz}$ to $65 \mathrm{GHz}$ with a mean antenna efficiency of $79 \%$.
\end{abstract}

Keywords: 3-D printing; Fresnel lens; millimeter-waves; lens antenna; polarization insensitivity

\section{Introduction}

Lens antennas are the most-promising antenna solutions for the future of wireless communications at millimeter-wave frequencies [1]. This kind of antennas allow to enhance low-directivity antennas such as open-ended waveguides or patch antennas [2]. The gain, which is closely related to the directivity, must be a large number at millimeter-waves communications to overcome the severe path loss introduced in the link budget of a wireless system.

Two main groups of lens antennas can be distinguished depending on the number of refractive indexes employed. On the one hand, there are the gradient-index (GRIN) lenses that require different refractive indexes in the lens structure $[3,4]$. On the other hand, there is the homogeneous lens that is implemented with a single refractive index value, but the shape of the lens defines its focusing performance [5].

In the design of lens antennas, the determination of the refractive indexes is crucial for a successful implementation of the lens. Due to the significant evolution of the 3-D-printing technologies and the availability of a wide range of materials with different permittivity, GRIN lenses based on 3-D printing have been reported in the literature. Among the GRIN lenses, Luneburg [6-8], Gutman [9], and flat lenses [10-12] are highlighted. Luneburg and Gutman lenses provide an ultra-wideband performance with low scanning losses. However, to further increment the directivity, a 2-D focusing is needed, but theses lenses become bulky with a non-planar profile. Transformation optics can be applied to achieve the desired planar profile, but unfortunately it introduces greater refractive index values in the lens structure $[7,12]$. The GRIN flat lenses inherently have a planar profile, but a 
larger aperture of this kind of lens requires either increasing the maximum refractive index or thickening the lens. This fact could be a drawback in any case of implementation or manufacturing.

Another type of inhomogeneous lens, in addition to the above mentioned, is the Fresnel lens (FL) [13]. At the expense of reducing the operating bandwidth, this kind of lens is also capable of producing a far-field focusing with a simple and low-profile design. One of the main advantages of the Fresnel lens design is the flexibility in the selection of the required permittivity values [14]. Moreover, there is no need to use matching layers (as commonly employed in GRIN flat lenses [15]) since the thickness of the Fresnel lens can be tuned to avoid reflections. Several FL designs based on 3-D printing have been reported. In [16], a classical grooved-dielectric Fresnel zone plate (FZP) was implemented with fused deposition modeling (FDM). An enhanced version of the FZP antenna designed by printed dielectric posts is presented in [17]. If the multidielectric zone version is selected instead of the grooved-dielectric FZP, more design control of the FL is achieved [14]. The lens antennas presented in [18-20] provide 3-D-printed FL designs in different operating frequency ranges and 3-D manufacturing. In contrast with the previous works, in this article is presented a cost-effective design of a 3-D-printed FL for the $60 \mathrm{GHz}$ band. The unit cells, which form the Fresnel lens, allow to be implemented in an easy manner since they can be oriented in any azimuth direction $\phi$ without changing its relative permittivity. This enhanced feature reduces the complexity in the implementation of the Fresnel subzones by means of 3-D-printed unit cells. The design of the unit cell provides insensitivity to TEM modes whose electric field points in either the vertical (TE polarization) or horizontal (TM polarization) direction. In addition, the geometry of the unit cell produces self-supporting subzones and effortless 3-D-printing fabrication with SLA.

This article is organized as follows. In Section 2, the proposed FL and unit-cell designs are analyzed by electromagnetic simulations. Section 3 discusses the manufacturing and measurements of the 3-D-printed FL. Finally, in Section 4, the main conclusions are presented.

\section{Fresnel Lens Design}

The proposed FL design and the chosen feeder is shown in Figure 1a. The lens is illuminated at a distance $F$ by an open-ended waveguide (OEWG) corresponding to a WR15 waveguide that represents a low-directivity radiator $(7.5 \mathrm{dBi}$ at $60 \mathrm{GHz})$. The FL is composed by three zones, each of them composed of four subzones; therefore, there are a total of 12 subzones. In Figure $1 \mathrm{~b}$ is indicated the subzones by a color coding (yellow, red, blue, and light blue), which is periodically repeated from the center of the lens up to the furthest subzone. The $n$-th subzone has an outer radius of $b_{n}$; as an example, the first zone of the lens will be described as follows. The first subzone is composed by four subzones, and these four subzones are repeated three times along the radius of the lens because the total number of zones implemented in the FL is three. The first subzone is highlighted in yellow at the lens center; the subzone radius is $b_{1}$; and its relative permittivity is $\varepsilon_{r 1}$. The circular ring next to the previous subzone is the second subzone that is highlighted in red whose radius is $b_{2}$, and its relative permittivity is $\varepsilon_{r 2}$. Then, the third subzone is highlighted in blue whose radius is $b_{3}$, and its relative permittivity is $\varepsilon_{r 3}$. Finally, the fourth subzone is highlighted in light blue whose radius is $b_{4}$, and its relative permittivity is $\varepsilon_{r 4}$. The values of the $b_{n}$ and the relative permittivities were selected according the design equations presented in [14]. Multidielectric FL provides a great flexibility in the design stage considering the maximum dielectric constant that can be achievable. In our case, the used resin for the SLA printing had a relative permittivity $\varepsilon_{r}$ of 2.6. Simply by modifying the thickness $t$ of the lens, a FL can be effectively designed where the required relative permittivities $\left(\varepsilon_{r 1}, \varepsilon_{r 2}, \varepsilon_{r 3}\right.$, and $\left.\varepsilon_{r 4}\right)$ are lower than the maximum value set for $\varepsilon_{r}$. Unlike other types of lenses, where their relative permittivity distribution is already established [7-9], the FL can be adjusted to the employed printed material. In Table 1 are listed the value of parameters used in the proposed FL design. The total diameter (D) of the 
lens was $71.4 \mathrm{~mm}$ indicated by the radius of the subzone furthest away from the center; this is $b_{12}$. The value for the thickness of the lens $t$ was selected to reduce undesired reflections in the first subzone $b_{1}$ [14], while the F/D ratio had been set to 0.448 in order to improve the lens directivity along the frequency range. This enhancement depends on the illumination provided by the OEWG and, in consequence, to the distance between the lens and its feeder (OEWG in our case).

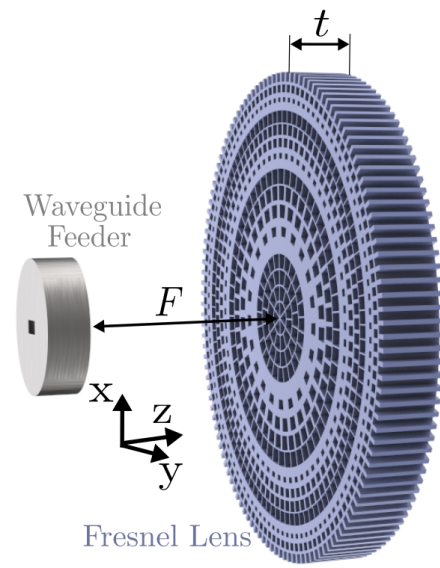

(a)

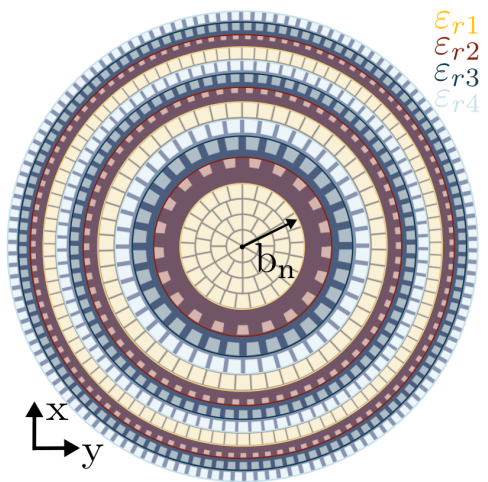

(b)

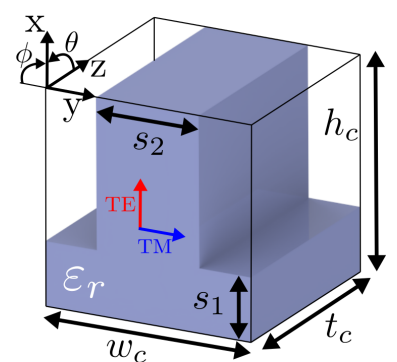

(c)

Figure 1. Fresnel lens design: (a) 3D view of the lens and the waveguide feeder. (b) Planar view of the subzones. (c) Dielectric unit cell with T-shaped geometry.

Table 1. Values (in $\mathrm{mm}$ ) of the parameters used in the design of the Fresnel lens.

\begin{tabular}{ccccccccccccc}
\hline Param. & $\boldsymbol{t}$ & $\boldsymbol{F}$ & $\boldsymbol{\varepsilon}_{\boldsymbol{r}}$ & $\boldsymbol{\varepsilon}_{\boldsymbol{r} \mathbf{1}}$ & $\boldsymbol{\varepsilon}_{\boldsymbol{r} \mathbf{2}}$ & $\boldsymbol{\varepsilon}_{\boldsymbol{r} \mathbf{3}}$ & $\boldsymbol{\varepsilon}_{\boldsymbol{r} \mathbf{4}}$ & $\boldsymbol{b}_{\mathbf{1}}$ & $\boldsymbol{b}_{\mathbf{2}}$ & $\boldsymbol{b}_{\mathbf{3}}$ & $\boldsymbol{b}_{\boldsymbol{4}}$ & $\boldsymbol{b}_{\mathbf{5}}$ \\
\hline Value & 8.94 & 32 & 2.6 & 1.25 & 2.36 & 1.95 & 1.58 & 9.4 & 13.5 & 16.6 & 19.4 & 21.8 \\
\hline Param. & $\boldsymbol{b}_{\boldsymbol{6}}$ & $\boldsymbol{b}_{\mathbf{7}}$ & $\boldsymbol{b}_{\mathbf{8}}$ & $\boldsymbol{b}_{\mathbf{9}}$ & $\boldsymbol{b}_{\mathbf{1 0}}$ & $\boldsymbol{b}_{\mathbf{1 1}}$ & $\boldsymbol{b}_{\mathbf{1 2}}$ & $\boldsymbol{s}_{\boldsymbol{b} \mathbf{1}}$ & $\boldsymbol{s}_{\boldsymbol{b} \mathbf{2}}$ & $\boldsymbol{s}_{\boldsymbol{b} \mathbf{3}}$ & $\boldsymbol{s}_{\boldsymbol{b} \mathbf{4}}$ & \\
\hline Value & 24.1 & 26.2 & 28.3 & 30.2 & 32.1 & 33.9 & 35.7 & 0.26 & 2.75 & 1.32 & 0.72 & \\
\hline
\end{tabular}

In Figure 1c, it is illustrated the design of the unit cell that forms the subzones of the FL. The unit cell presents a T-shaped geometry that is uniform along the propagation direction $z$. A typical strategy to reduce the relative permittivity of a dielectric cube is by inserting air zones in the unit-cell structure. In our case, rectangular air slabs are introduced symmetrically (uncolored zones) to allow a tuning of the relative permittivity. Modifying parameters $s_{1}$ and $s_{2}$ of the T-shaped geometry and keeping fixed the parameters $w_{c}$ and $h_{c}$, the relative permittivity of the unit cell can range from 1 to $\varepsilon_{r}$. In order to choose the values for $s_{1}$ and $s_{2}$, which determine the $\varepsilon_{r n}$ for each subzone, full-wave simulations in CST Microwave Studio were performed. Frequency-solver and unit-cell boundary conditions along the $x$ - and $y$-directions were imposed to properly compute the scattering parameters for the TE and TM polarizations. Then, the scattering parameters were analyzed through the formulation proposed in [21] to calculate the modified relative permittivity of the unit cell. It is important to note that the thickness $t_{c}$ of the unit cell does not affect the computed relative permittivity since there is no change in the structure along the propagation direction.

Since the dimensions of the T-shaped geometry alters the amount of air inside the unit cell, a filling factor $f$ was defined as the ratio between the area with dielectric and the total area in XY plane of the unit cell. Figure 2a shows the relative permittivities for different filling factors when the dimensions of the T-shaped geometry are equal $\left(s_{1}=s_{2}\right)$ and when they are not $\left(s_{1} \neq s_{2}\right)$ for a sub-wavelength unit cell. In this figure, the TE and TM polarizations are also assessed. It is important to note that any other incident linear polarization with different azimuth angle $\phi$ (see axis of Figure 1c) can be decomposed 
and analyzed by the orthogonal polarizations TE and TM analyzed in Figure 2a. It is observed the importance of the similarity in the dimensions of the T-shape to obtain a polarization-insensitive unit cell suitable for the radial arrangement in the proposed FL design. The reason for this fact is that both incident polarizations are affected by the same amount of dielectric material. The same conclusions can be extracted if the unit cell or the incident polarization is rotated in $\phi$. In this case, the impinging polarization can be decomposed in two vectors aligned with the edges of the unit cell that can be analyzed as TE and TM in Figure 1c. If the dimensions are not equal, the relative permittivity of the unit cell depends on the incident polarization, which results in a non-constant relative permittivity in the subzones of the FL (see Figure 2a). This fact entails that the relative permittivity of each subzone depends on the relative position between incident polarization and the orientation of the unit cell in the subzone. Figure $2 b$ shows the performance of unit cells with different permittivity values along the target frequency range for the case when $s_{1}=s_{2}$ with a normal incidence $\left(\theta=0^{\circ}\right)$. As it was expected because the unit cell is fully dielectric, almost-constant values for the dielectric constant were obtained for TE and TM polarizations, which is also desired for the FL design.

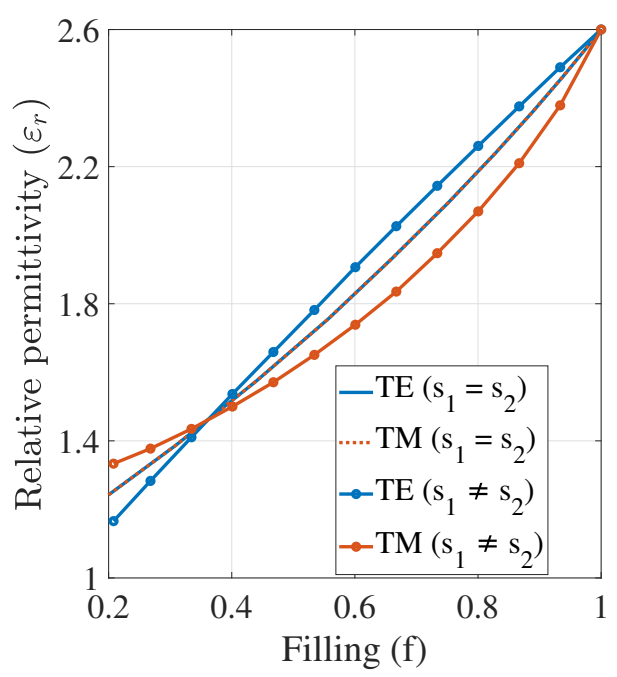

(a)

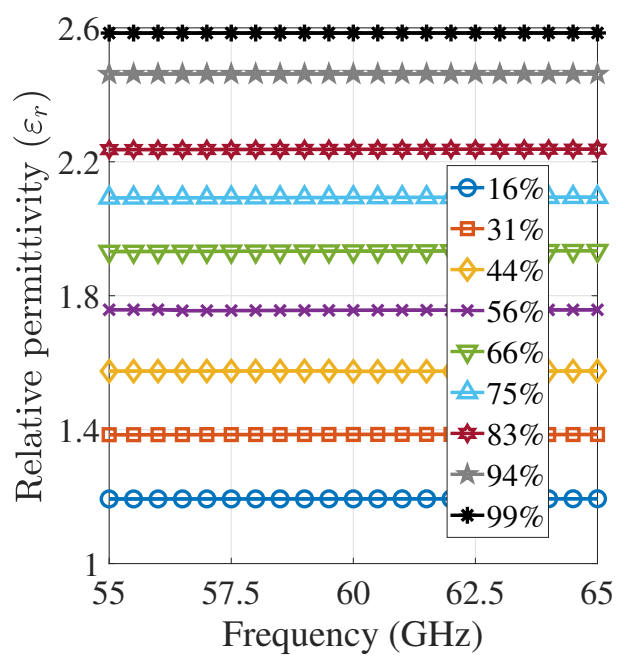

(b)

Figure 2. (a) Relative permittivity of the unit cell when the filling factor varies at $60 \mathrm{GHz}$. The dimensions are: $w_{c}=h_{c}=t_{c}=1 \mathrm{~mm}$. For the case $s_{1} \neq s_{2}, s_{2}=0.2 \mathrm{~mm}$ and $s_{1}$ varies. (b) Relative permittivity along the frequency for different filling factors.

The S-parameters in phase and magnitude of the unit cell with equal dimensions $\left(s_{1}=s_{2}\right)$ under oblique incidence was examined in Figure 3 . In this case, the thickness $t_{c}$ of the unit cell was set to $8.94 \mathrm{~mm}$, which is the total thickness of the FL (see Table 1), and dielectric losses $(\tan \delta=0.02)$ were also included to accurately evaluate the performance in the magnitude of the proposed unit cell. The maximum angle assessed for oblique incidence $(\theta)$ corresponds to the relative position between the waveguide feeder and the outermost Fresnel subzone. Figure 3 a shows the variation in the transmission phase between the normal incidence and the extreme oblique incidence $\left(\theta=48^{\circ}\right)$. The smaller the difference between the transmission-phase values obtained for normal incidence and oblique incidence, the less error is introduced in the Fresnel-lens-design equations (mainly Equation (3) from [14]). Thus, being farther away from the center subzones was found to provide the desired constructive interference at the focus of the lens. The transmission phase implicitly takes into account the relative permittivity produced by each oblique incidence angle. The transmission phase difference was at most $20^{\circ}$ for the entire fillingfactor range and both orthogonal polarizations. This performance was enabled by the T-shaped unit cell with equal dimensions. Moreover, due to the fully dielectric nature of 
the unit cell in which the dielectric material had a relatively low permittivity (2.6), possible resonances in the unit cell structure were avoided in the target frequency range for both normal and oblique incidence (up to $\theta=48^{\circ}$ ). These possible resonances that may appear at oblique incidence drastically modify the electromagnetic response observed at normal incidence. On the other hand, Figure $3 b, c$ present the transmission response in magnitude for the different relative permittivities and oblique incidence angles, respectively. The considered relative permittivities in these simulated results are the ones needed for the FL subzones. Figure $3 b$ shows transmission losses between $0.3 \mathrm{~dB}$ and $2 \mathrm{~dB}$ for the four required types of unit cell (one for each subzone). The losses observed were produced mainly by two factors: reflection in the dielectric-air interface of the unit cell and dielectric losses due to the loss tangent of the employed resin material. The unit cell with relative permittivity $\varepsilon_{r 1}$ is the one that produces lower losses because it has the closest relative permittivity (1.25) to that of the free space, and it has the lowest amount of dielectric material in its structure regarding the other types of unit cells. In contrast, a unit cell that provides the $\varepsilon_{r 2}$ has higher relative permittivity and contains more dielectric material in its structure. In consequence, it provides higher losses in the transmission coefficient as was expected for the two above factors. In Figure 3c, the transmission coefficient for different oblique incidence angles is assessed in the unit cell that provides $\varepsilon_{r 3}$. As the incidence angle increased, the transmission magnitude slightly varied with respect to the normal incidence case. Therefore, it was demonstrated that the proposed unit cell provides robustness to large oblique incidence angles beneficial for preserving the behavior of the subzones farthest from the center of the lens. This unit-cell characteristic was scarcely analyzed in previous 3-D-printed Fresnel lenses.

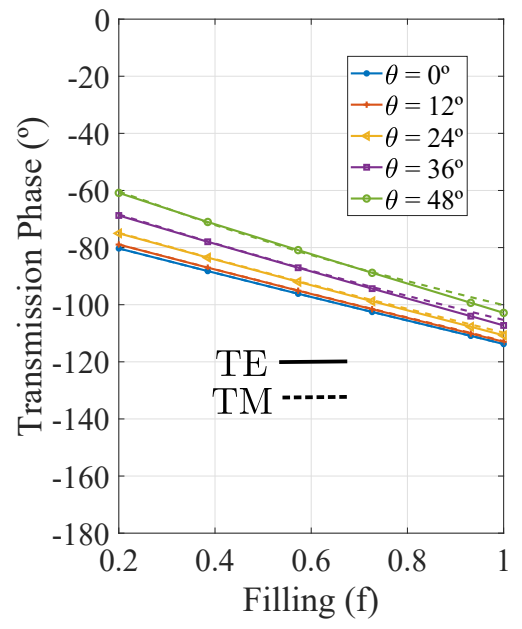

(a)

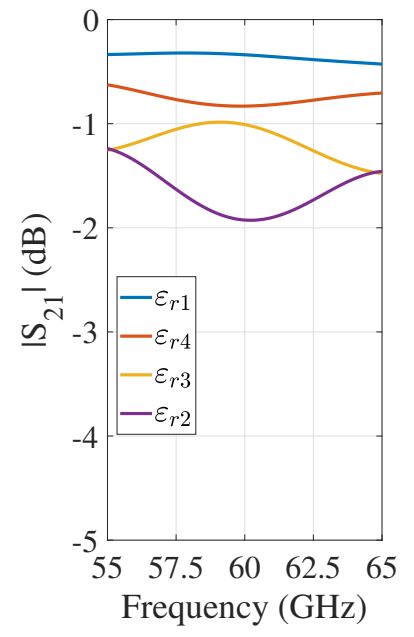

(b)

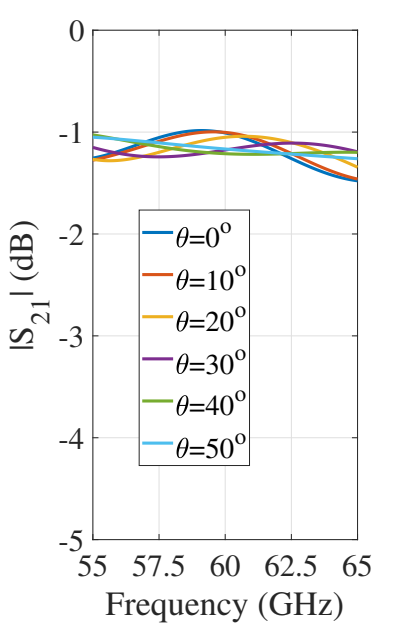

(c)

Figure 3. (a) Transmission phase response of the proposed unit cell for different filling factors, oblique incidence angles, and polarizations. The dimensions are: $w_{c}=h_{c}=1 \mathrm{~mm}, t_{c}=8.94 \mathrm{~mm}$, and $s_{1}=s_{2}$. (b) Transmission magnitude response for the relative permittivities required in the FL subzones under normal incidence. (c) Transmission magnitude response for the unit cell of the third subzone $\left(\varepsilon_{r 3}\right)$ under different oblique incidence angles.

In order to achieve the above required permittivities for the subzones, both dimensions $s_{1}$ and $s_{2}$ of the T-shaped geometry for each subzone are represented as $s_{b n}$ (see Figure $1 \mathrm{~b}$ ), $n$ being the number of the subzone. Table 1 includes the dimension $s_{b n}$ up to the fourth subzone. The dimensions for the other subzones can be calculated by means of a size scaling knowing the aspect relations of the first four subzones. This scaling process approximately keeps the designed relative permittivity as long as the unit cell is sub-wavelength. For the first subzone of the FL design, four unit cells were implemented, while in the other subzones, a single unit cell in the radial direction was only required. In this manner, 
the sizes of all the unit cells, which compose the FL, were sub-wavelength providing the designed relative permittivity. The unit cells were square and had equal dimensions for the T-shaped geometry to preserve the relative permittivity of the subzone under any polarization. This fact enable a cost-effective implementation of the FL subzones by the radial arrangement of the unit cells. Moreover, the proposed unit cell is suitable in the 3-D-printing process because all the subzones are self-supported, avoiding the inclusion of additional supports.

Figure $4 \mathrm{a}$ illustrates the phase and amplitude distributions of the electric field in the planes before (framed in gray) and after (framed in black) the FL antenna. The low directivity provided by the waveguide feeder produces a spherical wavefront that impinges in the lens. The FL transforms this spherical wavefront into a flatter wavefront over the entire transverse plane after the lens. Regarding the amplitude distribution in the planes under analysis (illustrated on the right side of the Figure 4a), the amplitude becomes more uniform across the lens due to the phase correction. However some variations in amplitude were still observed caused by the different transmission coefficients provided by the unit cells that form the subzones (Figure 3b). In spite of this fact, the field transformation in the phase wavefront produced by the FL allows a huge increase in directivity. Figure $4 \mathrm{~b}$ presents the E- and H-plane radiation-patterns comparison between the homogeneous multidielectric, which represents an ideal subzone implementation, and the proposed FL. Good agreement was observed in the comparison, which indicates the effectiveness of the proposed unit cells for the FL design.

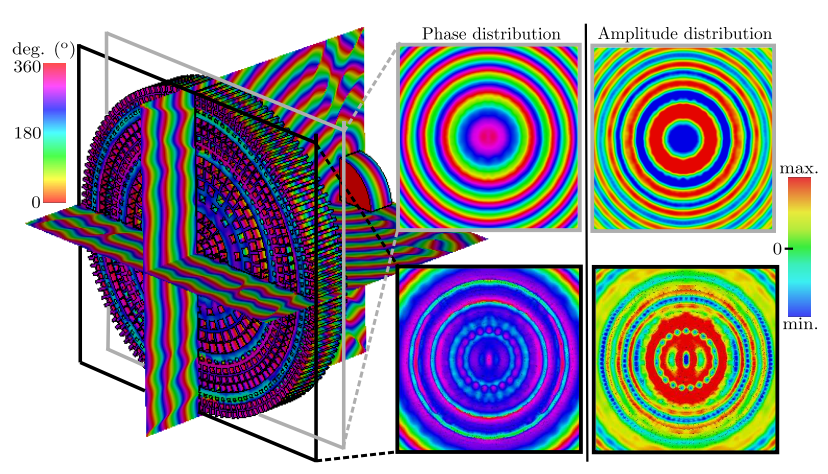

(a)

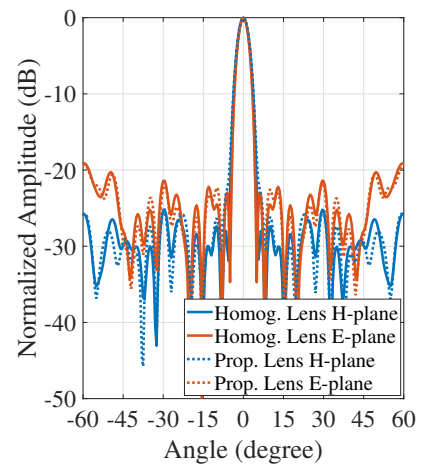

(b)

Figure 4. Radiation performance of the Fresnel-lens antenna: (a) Amplitude and phase distributions at $60 \mathrm{GHz}$. (b) Radiation-patterns comparison with the homogeneous multidielectric Fresnel lens at $60 \mathrm{GHz}$.

\section{Manufacturing and Measurements}

The depicted FL design was manufactured with SLA printing. The employed 3-D printer was the Form 3 from Formlabs, and the grey resin was printing material. A $50 \mu \mathrm{m}$ thickness layer with a horizontal orientation for the printed lens was adopted to achieve a successful printing. This orientation for the FL is very important to correctly print the unit cells where the air zones cannot be covered by resin [17]. Additionally, high vertical supports were included to conserve theses air zones during the printing process. Once the printing was finished, the lens was washed with isopropyl alcohol, cured, and sanded on the surface where the supports were located.

Far-field performance of the printed FL was measured in the anechoic chamber of the University of Granada. Figure 5a shows the measurement setup and the lens prototype. Both insets included in Figure 5a display the same printed Fresnel lens with different light illumination. An antenna holder (illustrated on the right side of the figure) was 3-D printed to align the lens with the OEWG and set the focal distance between them. In addition, the antenna holder contains fixings for moving the FL along its $y$-direction and, in this manner, assess its scanning performance. These positions are indicated in the figure as well 
as the OEWG (waveguide feeder). The distance between positions was $6 \mathrm{~mm}$, achieving an offset between the center of the Fresnel lens and the OEWG of $24 \mathrm{~mm}$ at the farthest position. The smaller the distance between positions, the higher the resolution of the beam-steering angle. The simulated and measured results for the reflection coefficient with and without the FL are shown in Figure $5 b$. The lens antenna system provides a good impedance matching (below $-10 \mathrm{~dB}$ ) along the entire frequency band both with and without the FL. This is because the reflection mainly occurs in the radiation of the OEWG. Some differences between simulated and measured results exist due to the use of a WR15 coax-to-waveguide transition that is necessary to connect the OEWG to the vector-network analyzer (VNA). It was not possible to include this transition in the calibration, but it does not alter the measurements since the measured reflection coefficient level is approximately the simulated one in the target frequency range.
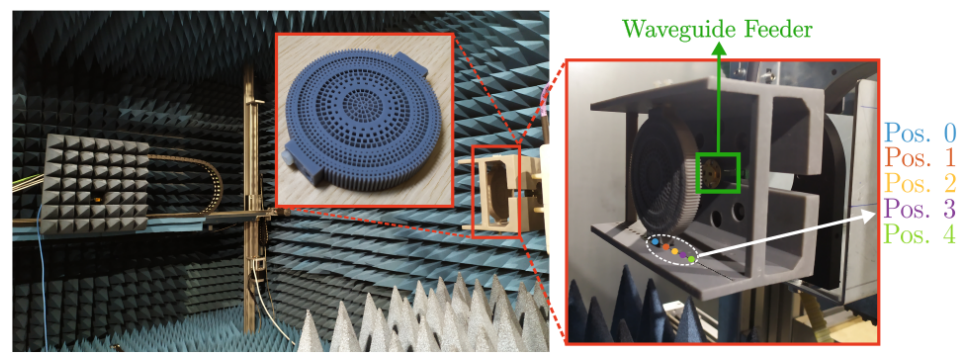

(a)

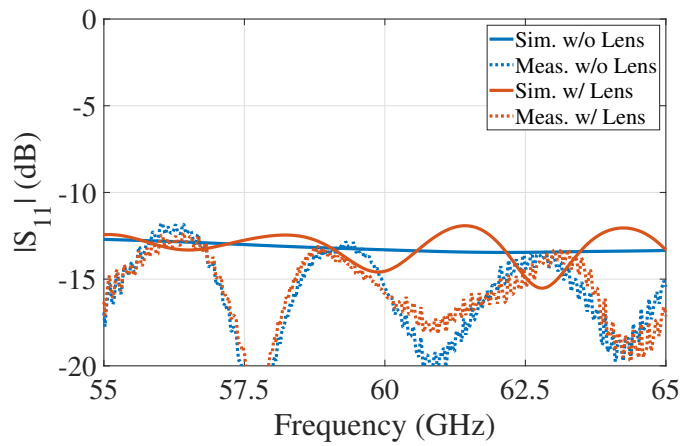

(b)

Figure 5. (a) 3-D printed prototype and far-field measurement setup. (b) Simulated and measured results of the Fresnel-lens antenna.

Figure 6 presents the simulated and measured radiations patterns for the E-plane (see Figure 6a) and H-plane at different positions and frequencies (see Figure $6 \mathrm{~b}-\mathrm{d}$ ). Position 0 corresponds when the OEWG is fully aligned with the FL. The rest of the positions represent a $6 \mathrm{~mm}$ movement of FL along its $y$-direction. The larger this movement is, the higher is the pointing angle of the main beam in the H-plane. The measurements reveal that the direction of the main beam is preserved over the frequency and in both planes. Moreover, the beamwidth narrows as the frequency increases, and thus the directivity increases. However, this beamwidth increases, and side lobes appear for larger pointing angles.

In Figure $7 \mathrm{a}$, the simulated and measured gains along the frequency range for the different positions of the lens are shown. It has been included the measured gain of the reference horn antenna (Flann WR15 Standard Gain Horn) used in the measurements to guarantee the correct calculation of the gains. The maximum gain achieved was $27 \mathrm{dBi}$ for the central position. The gain decreased as the pointing angle increased, which was expected from the simulated radiation patterns of Figure 6. For the farthest position (pos. 4), the gain was around $21 \mathrm{dBi}$, and the pointing angle was $33^{\circ}$. This implies scanning losses of $0.18 \mathrm{~dB} /{ }^{\circ}$, which are reasonable for this type of lens. In each measured position, the measured gain agrees with the simulated results, which proves the similarity between the estimated-loss tangent $(\tan \delta=0.02)$ and the experimental one. In addition, it is important 
to note that the beam-scanning capability of the Fresnel-lens design can be assessed because of the use of a low-directivity feeder. If a feeder with higher directivity had been employed instead, the maximum scanning angle would be smaller because the increase in the lens offset would mean that the lens would receive less power to be re-radiated. In the frequency range, the measured crosspolar level was under $-23 \mathrm{~dB}$ and the mean antenna efficiency (calculated as the ratio of the simulated directivity, and the measured gain) is $79 \%$. Besides, the aperture efficiency without including the antenna losses, which are taken into account in the antenna efficiency, was about $30 \%$ at $60 \mathrm{GHz}$, which was the design frequency. This value for the aperture efficiency was satisfactory for this kind of antenna system, but it can be improved by the use of a feeder with higher directivity such as a horn antenna. This inclusion would increase, for instance, the spillover efficiency, but also, it would significantly increase the cost of the whole antenna system. In addition, in Figure 7a, the agreement between the simulated and measured gain gets poorer at the end of the frequency range. This is mainly caused by the tolerances in fixing the focal distance $F$. In order to check the effect of this mechanical tolerance in the gain of the lens for Pos. 0 , Figure $7 \mathrm{~b}$ shows the performance of the simulated gain of the FL along the target frequency range when $F$ varies. As it can be observed, $F$ modifies the behavior of the provided gain along the frequency range. From this figure, we can deduce a lower value for the focal distance in the measurement setup since they are the values that produce a decrease in gain at the end of the measured frequency range. Besides this fact, Figure $7 \mathrm{~b}$ clearly shows the gain stability at the design frequency $(60 \mathrm{GHz})$ when the focal distance is modified and how the distance $F$ can be tuned to the gain values on both sides of the design frequency.

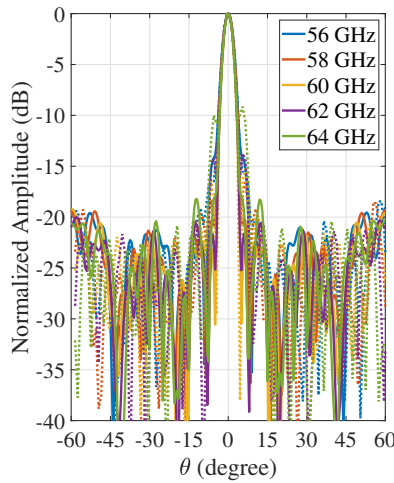

(a)

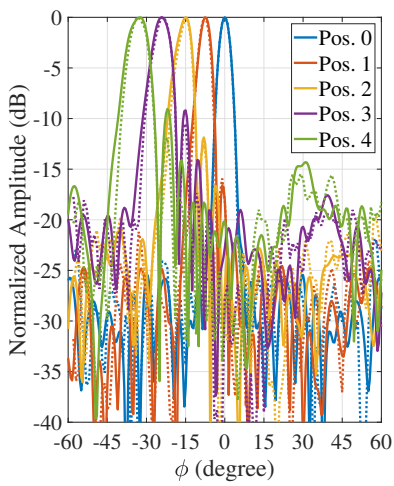

(c)

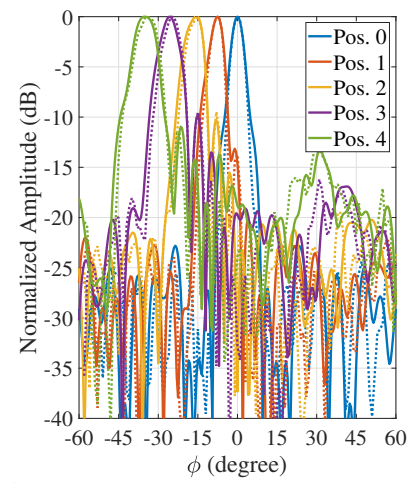

(b)

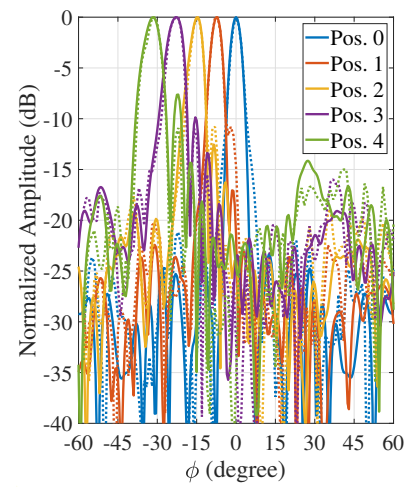

(d)

Figure 6. Normalized radiation patterns of the proposed Fresnel lens antenna: (a) E-plane for pos. 0. (b) H-plane at $56 \mathrm{GHz}$. (c) H-plane at $60 \mathrm{GHz}$. (d) H-plane at $64 \mathrm{GHz}$. The simulated and measured results are represented by solid and dashed lines, respectively. 


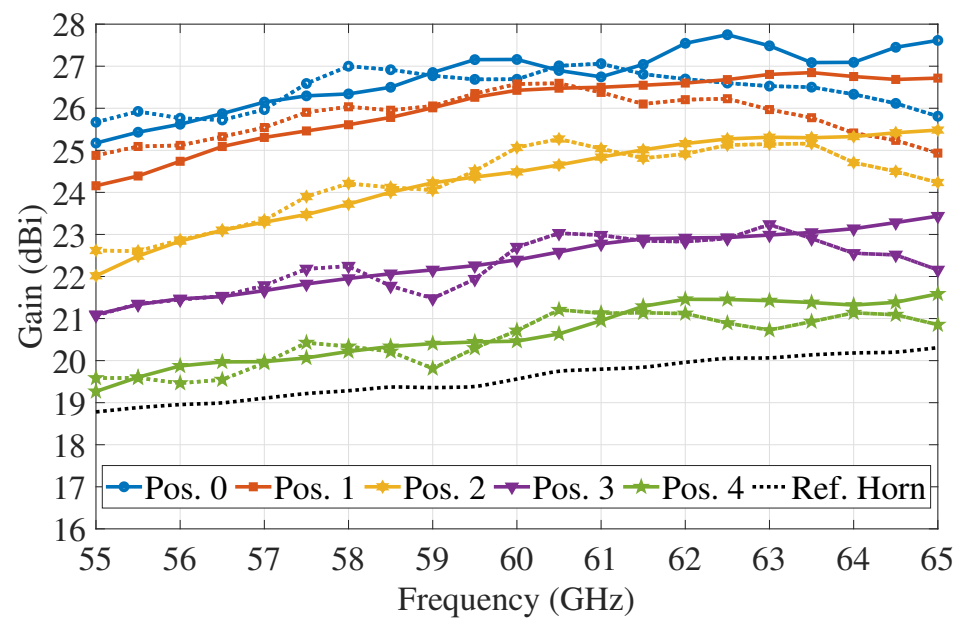

(a)

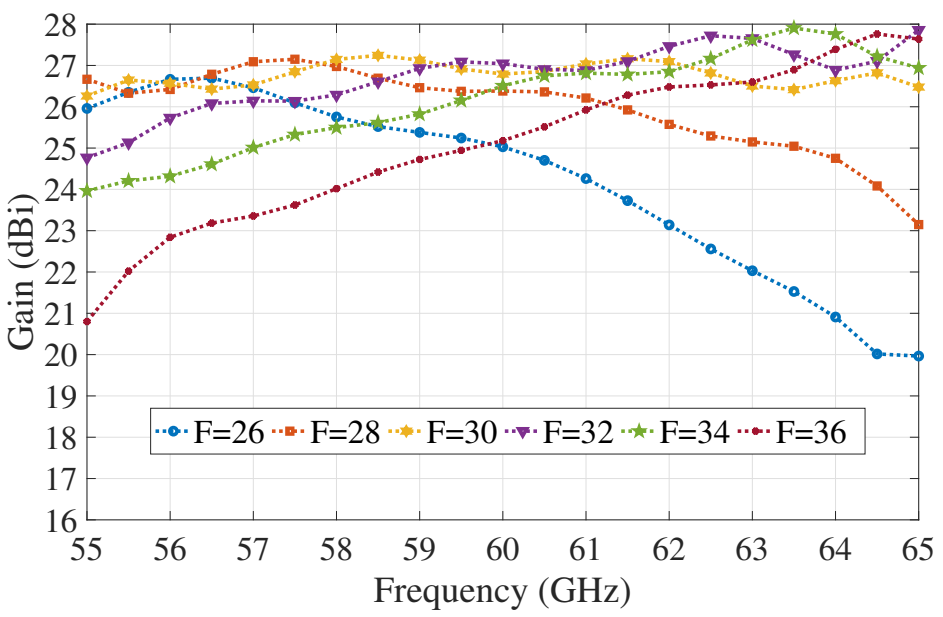

(b)

Figure 7. (a) Comparison of the gain along the frequency for different lens positions. The simulated and measured results are represented by solid and dashed lines, respectively. (b) Simulated gain along the frequency when the focal distance $F$ is modified.

Finally, in Table 2, the proposed FL design is compared with other 3-D-printed FL found in the literature. Our work presents the lowest maximum relative permittivity in the FL design achieving a similar maximum gain even using a low-cost feeder such as OEWG. Due to the geometry of the proposed unit cell, it can be implemented radially in the lens preserving the relative permittivity of the subzones under any incident polarization. In the related works $[19,20]$, their unit cells were analyzed, designed, and implemented in only one orientation, and thus the subzones implementation becomes more complex and limiting. The T-shaped geometry presents greater flexibility in the FL design and a self-supported implementation of the Fresnel subzones for 3-D printing manufacturing. Additionally, the T-shaped unit cell allows to implement unit cells with very low relative permittivity (1.25) without compromising the unit-cell printing and, thus, the lens fabrication. Scanning losses are also included in the comparison table; most of the reported works do not evaluate this radiation characteristic because the employed feeder has a directive radiation diagram, which poorly illuminates the Fresnel lens when the relative position between lens and radiator is varied. The work in [19] can assess the scanning losses because it uses an OEWG as the presented work. Since the same design procedure is followed for the Fresnel lens antenna, similar performance in the scan loss was obtained. 
Table 2. Comparison of reported 3-D printed Fresnel-lens antennas with this work.

\begin{tabular}{cccccccc}
\hline Ref. & $\begin{array}{c}\text { Freq. } \\
\mathbf{( G H z )}\end{array}$ & $\begin{array}{c}\text { 3-D-Printing } \\
\text { Technology }\end{array}$ & $\begin{array}{c}\text { Max. Gain } \\
\mathbf{( d B i )}\end{array}$ & Max. $\varepsilon_{r}$ & $\begin{array}{c}\text { Unit-Cell } \\
\text { Geometry }\end{array}$ & $\begin{array}{c}\text { Subzone } \\
\text { Implement. }\end{array}$ & $\begin{array}{c}\text { Scan Loss } \\
\left(\mathbf{d B} /{ }^{\circ}\right)\end{array}$ \\
\hline$[18]$ & 10 & FDM & 12.8 & 4.4 & Squared hole & Medium & n. a. \\
{$[16]$} & 30 & FDM & 24.6 & 2.86 & Homogeneous & Easy & n. a. \\
{$[19]$} & 22 & SLA & 27 & 3.06 & Squared hole & Medium & 0.16 \\
{$[20]$} & 60 & SLS & 28 & 3.6 & Cube shaped & Medium & n. a. \\
{$[17]$} & 300 & SLA & 27.4 & 2.66 & Dielectric post & Medium & n. a. \\
This Work & 60 & SLA & 27 & 2.6 & T-shaped & Easy & 0.18 \\
\hline
\end{tabular}

n. a.: not available.

\section{Conclusions}

In this study, a cost-effective design for a 3-D-printed Fresnel lens was proposed. The unit cell, which forms the subzones of the Fresnel lens, presents a T-shaped geometry that avoids the modification of the relative permittivity under TE and TM polarizations and oblique incidence. These features are highly desired to enable an effective implementation of the Fresnel subzones by means of a radial arrangement of the unit cell. To validate the proposed design, a 3-D-printed prototype was manufactured by SLA. The experimental results show an operating bandwidth from $55 \mathrm{GHz}$ to $65 \mathrm{GHz}$ where the maximum gain was $27 \mathrm{dBi}$. Additionally, due to the low-directivity radiator used as the feeder of the lens, the beam-steering capability was produced only by modifying the relative position between the Fresnel lens and the feeder. This radiation feature is of great interest in communications links at millimeter frequencies.

Author Contributions: Conceptualization, Á.P.-C.; methodology, Á.P.-C. and S.M.-R.; formal analysis and investigation, S.M.-R. and M.A.B.-M.; writing-original draft preparation, Á.P.-C., J.C.-M., S.M.-R. and M.A.B.-M.; writing-review and editing, Á.P.-C., J.C.-M., S.M.-R. and M.A.B.-M. All authors have read and agreed to the published version of the manuscript.

Funding: This work was supported in part by the Spanish Government under Project PID2020112545RB-C54; in part by "Junta de Andalucía" under Project B-TIC-402-UGR18, Project A-TIC-608UGR20, Project P18.RT.4830, and Project PYC20-RE-012-UGR; in part by the European Regional Development Fund and Junta de Extremadura under Project IB18003; and in part by the Predoctoral Grant FPU18/01965.

Data Availability Statement: Not applicable.

Conflicts of Interest: The authors declare no conflict of interest.

\section{References}

1. Quevedo-Teruel, O.; Ebrahimpouri, M.; Ghasemifard, F. Lens Antennas for 5G Communications Systems. IEEE Commun. Mag. 2018, 56, 36-41. [CrossRef]

2. Liu, K.; Zhao, C.; Qu, S.W.; Chen, Y.; Hu, J.; Yang, S. A 3-D-Printed Multibeam Spherical Lens Antenna with Ultrawide-Angle Coverage. IEEE Antennas Wirel. Propag. Lett. 2021, 20, 411-415. [CrossRef]

3. Ma, H.F.; Cui, T. Three-dimensional broadband and broad-angle transformation-optics lens. Nat. Commun. 2010, 1, 124, [CrossRef] [PubMed]

4. Jo, E.S.; Kim, D. 3-D Printer Based Lens Design Method for Integrated Lens Antennas. IEEE Antennas Wirel. Propag. Lett. 2018, 17, 2090-2093. [CrossRef]

5. Fernandes, C.A.; Lima, E.B.; Costa, J.R. Dielectric Lens Antennas. In Handbook of Antenna Technologies; Springer: Singapore, 2016; pp. 1001-1064. [CrossRef]

6. Wang, C.; Wu, J.; Guo, Y.X. A 3-D-Printed Wideband Circularly Polarized Parallel-Plate Luneburg Lens Antenna. IEEE Trans. Antennas Propag. 2020, 68, 4944-4949. [CrossRef]

7. Lou, Y.H.; Zhu, Y.X.; Fan, G.F.; Lei, W.; Lu, W.Z.; Wang, X.C. Design of Ku-Band Flat Luneburg Lens Using Ceramic 3-D Printing. IEEE Antennas Wirel. Propag. Lett. 2021, 20, 234-238. [CrossRef]

8. Lei, S.; Han, K.; Li, X.; Wei, G. A Design of Broadband 3-D-Printed Circularly Polarized Spherical Luneburg Lens Antenna for X-Band. IEEE Antennas Wirel. Propag. Lett. 2021, 20, 528-532. [CrossRef] 
9. Bjorkqvist, O.; Zetterstrom, O.; Quevedo-Teruel, O. Additive manufactured dielectric Gutman lens. Electron. Lett. 2019, 55, 1318-1320. [CrossRef]

10. Garcia-Marin, E.; Filipovic, D.; Masa-Campos, J.; Sanchez-Olivares, P. Low-cost lens antenna for 5G multi-beam communication. Microw. Opt. Technol. Lett. 2020, 62, 3611-3622. [CrossRef]

11. Imbert, M.; Papió, A.; De Flaviis, F.; Jofre, L.; Romeu, J. Design and Performance Evaluation of a Dielectric Flat Lens Antenna for Millimeter-Wave Applications. IEEE Antennas Wirel. Propag. Lett. 2015, 14, 342-345. [CrossRef]

12. Poyanco, J.M.; Pizarro, F.; Rajo-Iglesias, E. Wideband hyperbolic flat lens in the Ka-band based on 3D-printing and transformation optics. Appl. Phys. Lett. 2021, 118, 123503. [CrossRef]

13. Hristov, H.D. Fresnel Zones in Wireless Links, Zone Plate Lenses and Antennas, 1st ed.; Artech House, Inc.: Norwood, MA, USA, 2000.

14. Hristov, H.D.; Rodriguez, J.M. Design Equation for Multidielectric Fresnel Zone Plate Lens. IEEE Microw. Wirel. Components Lett. 2012, 22, 574-576. [CrossRef]

15. He, Y.; Eleftheriades, G.V. Matched, Low-Loss, and Wideband Graded-Index Flat Lenses for Millimeter-Wave Applications. IEEE Trans. Antennas Propag. 2018, 66, 1114-1123. [CrossRef]

16. Jeong, K.H.; Ghalichechian, N. Design, Fabrication and Measurement of a Millimeter Wave Fresnel Lens using Additive Manufacturing. In Proceedings of the 2018 IEEE International Symposium on Antennas and Propagation USNC/URSI National Radio Science Meeting, Boston, MA, USA, 8-13 July 2018; pp. 1879-1880. [CrossRef]

17. Wu, G.B.; Zeng, Y.S.; Chan, K.F.; Qu, S.W.; Chan, C.H. 3-D Printed Circularly Polarized Modified Fresnel Lens Operating at Terahertz Frequencies. IEEE Trans. Antennas Propag. 2019, 67, 4429-4437. [CrossRef]

18. Zhang, S. Design and Fabrication of 3D-Printed Planar Fresnel Zone Plate Lens. Electron. Lett. 2016, 52, 833-835. [CrossRef]

19. Monkevich, J.M.; Le Sage, G.P. Design and Fabrication of a Custom-Dielectric Fresnel Multi-Zone Plate Lens Antenna Using Additive Manufacturing Techniques. IEEE Access 2019, 7, 61452-61460. [CrossRef]

20. Pourahmadazar, J.; Denidni, T. Towards Millimeter-wavelength: Transmission-Mode Fresnel-Zone Plate Lens Antennas using Plastic Material Porosity Control in Homogeneous Medium. Sci. Rep. 2018, 8, 5300. [CrossRef] [PubMed]

21. Chen, X.; Grzegorczyk, T.M.; Wu, B.I.; Pacheco, J.; Kong, J.A. Robust method to retrieve the constitutive effective parameters of metamaterials. Phys. Rev. E 2004, 70, 016608. [CrossRef] [PubMed] 